tigators injected a single, large dose of an autoantibody that recognizes mouse rbcs to generate their cross-talk model system. The quantity of antibody used decreased the hematocrit from $50 \%$ to $25 \%$ in 4 days, even in $\mathrm{Fc} \gamma \mathrm{R}^{-/-}$animals. Thus, $\mathrm{C}$ activation and rbc destruction took place via intravascular lysis and receptor-mediated extravascular sequestration (7). Complement activation occurs rapidly (within seconds) in this model and would provide another source of $\mathrm{C} 5 \mathrm{a}$ to enhance $\mathrm{Fc} \gamma \mathrm{R}$ mRNA expression; this may be the most likely C5a source in vivo. Second, the authors do not establish the mechanism for generating $\mathrm{C} 5 \mathrm{a}$. They hypothesize that a protease cleaves C5. Third, while they demonstrate upregulation of $\mathrm{Fc} \gamma \mathrm{RI}$ and $\mathrm{Fc} \gamma \mathrm{RIII}$ mRNA expression, they do not document an increased number of $\mathrm{F} c \gamma$ Rs on Kupffer cells in vitro or in vivo. Fourth, the authors nicely establish that Fc $\gamma \mathrm{RII}$ (the Fc $\gamma \mathrm{R}$ that sends a negative or antiinflammatory signal) plays no role in their observations. The reason here is simple: Kupffer cells do not express this receptor, although Fcy RII was expressed on sinusoidal cells.

\section{Immunopathologic reactions}

As a final comment, in their study, Kumar et al. (1) refer to their model as an anti- body-dependent type II autoimmune reaction. Antibodies of this type are directed at cell-bound targets (in the case of autoimmune hemolytic anemia) or matrix proteins (in the case of Goodpasture syndrome). The most up-to-date immunology textbooks still all have tables showing the Gell and Coombs classification system of immunopathologic reactions (8). I first learned about this system, as did many aspiring immunologists of my generation, at the $\mathrm{NIH}$, where we were so fortunate to have Bill Paul as our teacher in the immunology course. I have now been teaching this same classification system for 30-plus years to students at Washington University School of Medicine. Students and senior immunologists still find it a useful scheme to classify immune reactions that we would like to be able to better control. Hopefully, insights provided by studies like those of Gessner's group will eventually provide us with the tools to accomplish this goal.

\section{Acknowledgments}

The author thanks Kathy Liszewski, Anna Richards, and David Kavanagh for their helpful review of this commentary, and Madonna Bogacki for secretarial assistance.
Address correspondence to: John P. Atkinson, Washington University School of Medicine, 660 South Euclid Avenue, Campus Box 8045, St. Louis, Missouri 63110-1093, USA. Phone: (314) 362-8391; Fax: (314) 362-1366; E-mail: jatkinso@im.wustl.edu.

1. Kumar, V., et al. 2006. Cell-derived anaphylatoxins as key mediators of antibody-dependent type II autoimmunity in mice. J. Clin. Invest. 116:512-520. doi:10.1172/JCI25336.

2. Shushakova, N., et al. 2002. C5a anaphylatoxin is a major regulator of activating versus inhibitory $\mathrm{F} c \gamma$ receptors in immune complex-induced lung disease. J. Clin. Invest. 110:1823-1830. doi:10.1172/ JCI200216577.

3. Godau, J., et al. 2004. C5a initiates the inflammatory cascade in immune complex peritonitis. J. Immunol. 173:3437-3445.

4. Schmidt, R.E., and Gessner, J.E. 2005. Fc receptors and their interaction with complement in autoimmunity. Immunol. Lett. 100:56-67.

5. Ravetch, J.V. 2002. A full complement of receptors in immune complex diseases. J. Clin. Invest. 110:1759-1761. doi:10.1172/JCI200217349.

6. Atkinson, J.P., and Frank, M.M. 1974. The effect of Bacillus Calmette-Guerin-induced macrophage activation on the in vivo clearance of sensitized erythrocytes. J. Clin. Invest. 53:1742-1749.

7. Azeredo da Silveira, S., et al. 2002. Complement activation selectively potentiates the pathogenicity of the IgG2b and IgG3 isotypes of a high affinity antierythrocyte autoantibody. J. Exp. Med. 195:665-672.

8. Janeway, C.J., Travers, P., Walport, M.J., and Shlomchik, M.J. 2005. Immunobiology: the immune system in health and disease. Garland Science, Taylor \& Francis Group. New York, New York, USA. 823 pp.

\title{
Mucus in chronic airway diseases: sorting out the sticky details
}

\author{
Lauren Cohn
}

Section of Pulmonary and Critical Care Medicine, Yale University School of Medicine, New Haven, Connecticut, USA.

\begin{abstract}
Mucous hypersecretion is a major cause of airway obstruction in asthma, chronic obstructive pulmonary disease, and cystic fibrosis. EGFR ligands and IL-13 are known to stimulate mucous induction, but the detailed mechanisms of epithelial mucous regulation have not been well defined. In this issue of the JCI, Tyner et al. show, in a mouse model of chronic mucous hypersecretion, that ciliated epithelial cell apoptosis is inhibited by EGFR activation, allowing IL-13 to stimulate the differentiation of these cells into goblet cells, which secrete mucus (see the related article beginning on page $309)$. In defining this coordinated, 2 -step process, we can consider the therapeutic effects of blocking mucous production. This begs the question, Is it possible to reduce airway obstruction in chronic lung disease by inhibiting EGFR activation and/or by inhibiting IL-13?
\end{abstract}

Nonstandard abbreviations used: COPD, chronic obstructive pulmonary disease.

Conflict of interest: The author has declared that no conflict of interest exists.

Citation for this article: J. Clin. Invest. 116:306-308 (2006). doi:10.1172/JCI27690.
In the respiratory tract, mucus is a critical component of the innate host defense system. On the airway epithelial cell surface, the sticky gel layer traps particles and the sol layer, which is predominantly water, contacts the surface of ciliated cells and permits moving of the gel out of the lower airways like an escalator so that it can ultimately be cleared by coughing or swallowing. Mucus also contains antibacterial agents to aid in its defense function. Pathogens and harmless proteins we inhale are thus removed from the respiratory tract and have a limited encounter with other immune components. In the bronchial airways, mucus is produced by surface epithelial cells with secretory features and a classical goblet shape, called goblet cells. Goblet cells produce mucins that are complexed with water in secretory granules and are released into the airway lumen. In the large airways, mucus is also produced by mucous glands. Under basal conditions, the columnar epithelial surface comprises a small percentage of goblet cells and a majority of ciliated cells. This struc- 


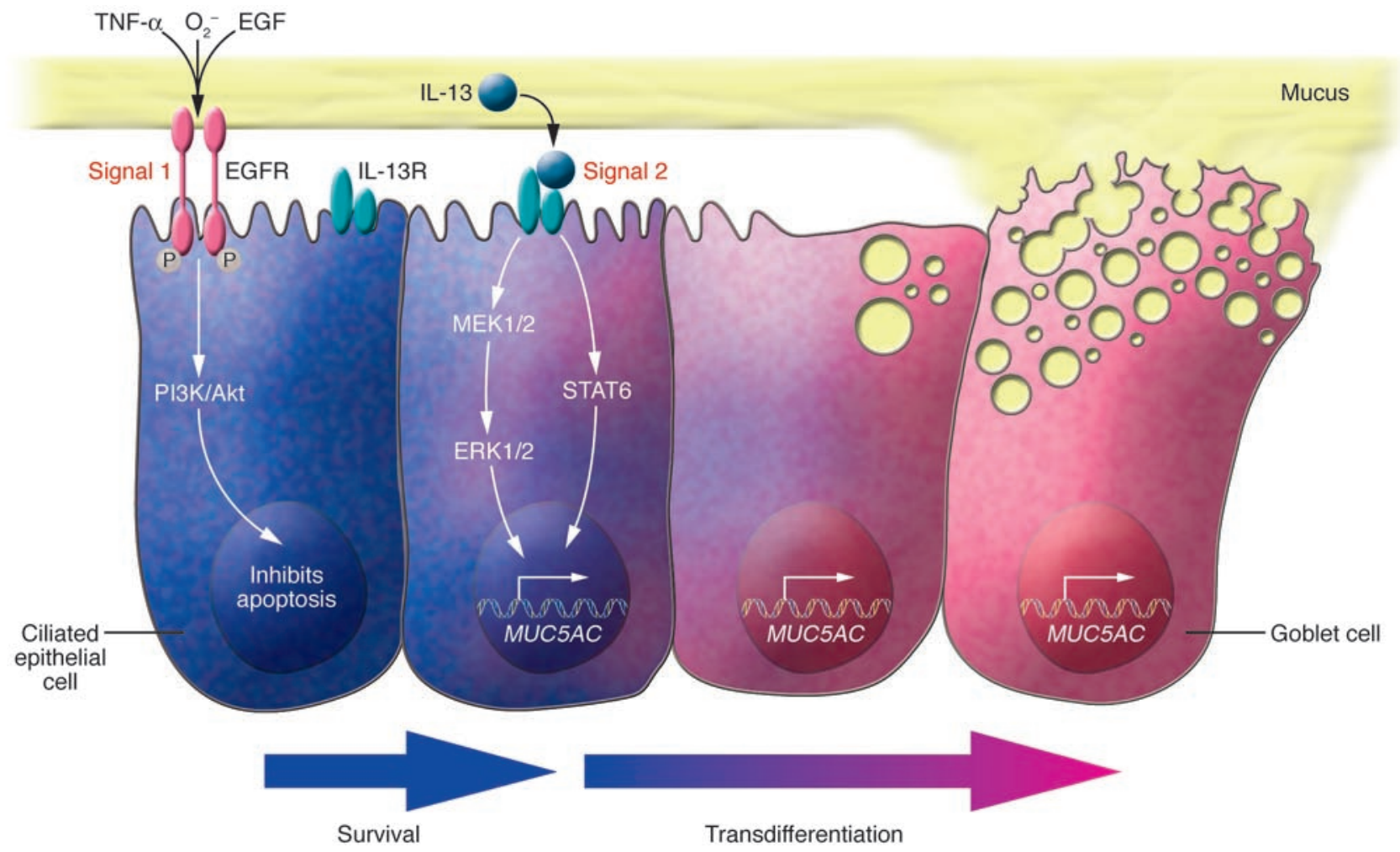

Figure 1

Ciliated cell differentiation into goblet cells requires 2 signals. Tyner et al. (7), using a mouse model of Sendai virus infection, propose that the induction of a chronic mucus-producing airway epithelium requires 2 signals. Signal 1 activates the EGFR on ciliated cells and induces EGFR phosphorylation and activation of PI3K/Akt. This pathway leads to inhibition of ciliated cell apoptosis. Ciliated cells that survive can respond to signal 2: IL-13 binding to its receptor. Upon IL-13 receptor (IL-13R) activation and STAT6 signaling, ciliated cells begin to produce mucins (including those encoded by MUC5AC), which are contained within mucous secretions, and lose their ciliated cell surface, taking on features of mucus-producing goblet cells. It also appears that other epithelial cells, such as Clara cells, can differentiate into goblet cells. Thus, the airway epithelium is driven to become a mucus-producing organ, presumably to enhance host defense. In some diseases, such as asthma, this response may be misdirected. Airway tissue from human asthmatics exhibits EGFR activation on ciliated cells, and mucus appears to be induced by IL-13, suggesting that this may also be an important pathway for mucous induction in humans, yet it remains unclear whether other pathways of mucous induction are active in chronic airway diseases.

ture provides adequate mucus to capture particles and remove them in the huge volumes of air we breathe. After infection or toxic exposure, the airway epithelium upregulates its mucous secretory ability, and we cough and bring up sputum. Subsequently, the airway epithelium recovers and returns to its normal state, goblet cells disappear, and coughing abates.

Mucous hypersecretion is a hallmark of chronic airway diseases, including asthma, chronic obstructive pulmonary disease (COPD), and cystic fibrosis, and goblet cell hyperplasia and persistence are characteristic pathologic features. In asthmatics, 20-25\% of airway epithelial cells are goblet cells, even in mild disease $(1,2)$. All of these diseases have distinct etiologies and different inflammatory responses that drive mucous hypersecretion. In asthma, inflammation appears to be mediated by allergen-specific Th2 cells, leading to eosinophilia, while in COPD, the inflammatory response is neutrophilic and may be induced by infection or components in cigarette smoke (3). Controlling inflammation is at the root of treatment through the use of corticosteroids and/or antibiotics, yet despite therapy, airway obstruction remains the cause of morbidity and mortality. Mucous secretions in the airways in asthma and COPD appear to be a major cause of airway obstruction, ventilation-perfusion mismatching, and hypoxemia, leading to wheezing and dyspnea (4-6). Can and should we be doing more to control mucus?

\section{Two-step model that leads to chronic mucous production}

In this issue of the JCI, Tyner et al. present a novel mechanism of chronic mucous hypersecretion in an asthma-like model and show a coordinated sequence of events that controls a conversion of normal epithelium into a chronic mucous secretory organ (7).
This progression clarifies how blockade of certain pathways might affect mucous production. Past studies by other groups identified 2 cytokine-mediated mechanisms of mucous induction, 1 activated by engagement of EGFR and the other dependent on IL-13 and STAT6 signaling (8-12). Tyner et al. (7) show for the first time that both of these signals are necessary for mucous production to occur. EGFR phosphorylation on ciliated cells inhibits apoptosis, and this allows the second signal, IL-13, to stimulate ciliated cells to differentiate into goblet cells (Figure 1). This logical sequence of events ensures that barrier function is maintained after an insult to the airway. Multiple ligands released after airway damage, including EGF, TNF- $\alpha$, and reactive oxygen species (13), activate the promiscuous EGFR. If an appropriate signal, such as IL-13, is provided, the epithelium can be converted to a mucus-producing organ that 
will sweep away pathogens and debris. Goblet cell differentiation does not occur in all immune responses, particularly those rich in IFN- $\gamma(14)$, so it appears to be closely regulated, yet the need for regulating mucous secretion is not clear.

In the model presented here (7), chronic mucous production follows Sendai virus infection in mice after viral clearance due to constitutive activation of EGFR in the absence of obvious inflammation. This effect is unique to 1 strain of mice and suggests a strain-specific response to the virus that leads to constitutive EGFR phosphorylation. After Sendai virus infection, mice exhibit an "abnormally programmed" epithelium, while in human airway diseases, mucous hypersecretion is most likely a normal response of the epithelium to inflammation. In these patients, airway inflammation provides an abundance of EGFR ligands to turn on $\operatorname{EGFR}(15,16)$.

\section{IL-13 stimulates mucus in asthma and possibly other airway diseases}

IL-13 is a potent stimulator of mucus in vivo, and its effects extend beyond the classical Th2 lymphocyte response. After Sendai virus infection, mucous hyperproduction is driven by IL-13, and this occurs in the absence of a visible airway inflammatory response. Likewise, mucous production is IL-13 dependent in non-Th2 driven airway inflammation when IL-13 levels are very low in the airways (17). Furthermore, many cytokine-driven models of airway inflammation result in mucous hypersecretion, yet each has been shown to do this through the production of IL-13 (18). Other factors can also induce mucus. For example, LPS can stimulate low-level mucous production in IL-13-deficient mice (17), and many studies have shown a role for human neutrophil elastase in mucous production $(19,20)$. Even in light of the seminal studies of mucin gene expression by Carol Basbaum $(21,22)$, there is only a rudimentary understanding of how these mediators stimulate mucins, the contribution of different mucins to the protective response, and which pathways are active in human disease. IL-13 is likely to play a critical role in mucous induction in asthma, and it may prove to be an important stimulus for mucous production in other chronic airway diseases, despite their diverse inflammation profiles, as IL-13 levels in the respiratory tract are often elevated $(23,24)$. In addition to its role in mucin gene expression, IL-13 induces other components of the secretory machinery, further supporting its identification as a master regulator of the goblet cell (25). Other candidates for the second stimulus have not been studied in such depth. They are lined up, and it is hoped they will be tested in this model by the Tyner and Holtzman laboratory.

\section{Does inhibition of mucus lead to clinical improvement in airway diseases?}

Inflammation in asthma results from an exuberant inflammatory response to allergens that pose no threat to the individual; therefore, reducing mucous production in response to these agents should improve patient symptoms. Blockade of mucus may be problematic if its overproduction facilitates removal of damaged cells. Inhaled steroids are effective in quelling both inflammation and mucous production in asthma, but even during this treatment, some inflammation persists, and when disease exacerbations occur, there is a swift increase in mucus. Clinical trials of inhibitors of IL-13, TNF- $\alpha$, and EGFR offer potential solutions to control airway obstruction and are on the horizon. Blocking the production of mucus in COPD has more potential to be detrimental since normal mucus helps to eliminate bacteria from the airways. It is unknown whether the mucus in these distorted airways inhibits or even promotes bacterial growth. Given the high prevalence of COPD, it may be worth defining a balance point that separates effective and pathologic mucous production. Since inhaled steroids have limited effectiveness in chronic bronchitis, therapeutic targeting of IL-13, TNF- $\alpha$, and EGFR offers a potential way to limit mucous production and improve lung function.

\section{Acknowledgements}

The author thanks Robert Homer and Donna Farber for helpful discussion. This work is supported by NIH grant NHLBI-64040.

Address correspondence to: Lauren Cohn, Yale University School of Medicine, 333 Cedar Street, P.O. Box 208057, New Haven, Connecticut 06520-8057, USA. Phone: (203) 737-1459; Fax: (203) 785-3826; E-mail: lauren.cohn@yale.edu.

\footnotetext{
1. Shimura, S., Andoh, Y., Haraguchi, M., and Shirato, K. 1996. Continuity of airway goblet cells and intraluminal mucus in the airways of patients with bronchial asthma. Eur. Respir. J. 9:1395-1401.

2. Rogers, D.F. 1994. Airway goblet cells: responsive and adaptable front-line defenders. Eur. Respir. J. 7:1690-1706

3. Barnes, P.J., Shapiro, S.D., and Pauwels, R.A. 2003.
}

Chronic obstructive pulmonary disease: molecular and cellular mechanisms. Eur. Respir. J. 22:672-688.

4. Hogg, J.C., Macklem, P.T., and Thurlbeck, W.M. 1968. Site and nature of airway obstruction in chronic obstructive lung disease. N. Engl. J. Med. 278:1355-1360.

5. Moreno, R.H., Hogg, J.C., and Pare, P.D. 1986. Mechanics of airway narrowing. Am. Rev. Respir. Dis. 133:1171-1180.

6. James, A., and Carroll, N. 1995. Theoretical effects of mucus gland discharge on airway resistance in asthma. Chest. 107:110S

7. Tyner, J.W., et al. 2006. Blocking airway mucous cell metaplasia by inhibiting EGFR antiapoptosis and IL-13 transdifferentiation signals. J. Clin. Invest. 116:309-321. doi:10.1172/JCI25167.

8. Guzman, K., Randell, S.H., and Nettesheim, P. 1995. Epidermal growth factor regulates expression of the mucous phenotype of rat tracheal epithelial cells. Biochem. Biophys. Res. Commun. 217:412-418.

9. Takeyama, K., et al. 1999. Epidermal growth factor system regulates mucin production in airways. Proc. Natl. Acad. Sci. U. S. A. 96:3081-3086.

10. Wills-Karp, M., et al. 1998. Interleukin-13: central mediator of allergic asthma. Science. 282:2258-2261.

11. Grunig, G., et al. 1998. Requirement for IL-13 independently of IL-4 in experimental asthma. Science. 282:2261-2263.

12. Cohn, L., et al. 1999. Th2-induced airway mucus production is dependent on IL-4Ralpha, but not on eosinophils. J. Immunol. 162:6178-6183.

13. Burgel, P.R., and Nadel, J.A. 2004. Roles of epidermal growth factor receptor activation in epithelial cell repair and mucin production in airway epithelium. Thorax. 59:992-996.

14. Cohn, L., Homer, R.J., Niu, N., and Bottomly, K. 1999. T helper 1 cells and interferon gamma regulate allergic airway inflammation and mucus production. J. Exp. Med. 190:1309-1318.

15. Chung, K.F. 2001. Cytokines in chronic obstructive pulmonary disease. Eur. Respir. J. Suppl. 34:50s-59s.

16. Holgate, S.T., Lackie, P.M., Davies, D.E., Roche, W.R., and Walls, A.F. 1999. The bronchial epithelium as a key regulator of airway inflammation and remodelling in asthma. Clin. Exp. Allergy. 29(Suppl. 2):90-95.

17. Whittaker, L., et al. 2002. Interleukin-13 mediates a fundamental pathway for airway epithelial mucus induced by CD4 T cells and interleukin-9. Am. J. Respir. Cell Mol. Biol. 27:593-602.

18. Cohn, L., Elias, J.A., and Chupp, G.L. 2004. Asthma: mechanisms of disease persistence and progression. Annu. Rev. Immunol. 22:789-815.

19. Park, J.A., et al. 2005. Human neutrophil elastase induces hypersecretion of mucin from well-differentiated human bronchial epithelial cells in vitro via a protein kinase Cdelta-mediated mechanism. Am. J. Pathol. 167:651-661.

20. Agusti, C., et al. 1998. Goblet cell degranulation after antigen challenge in sensitized guinea pigs. Role of neutrophils. Am. J. Respir. Crit. Care Med. 158:1253-1258.

21. Longphre, M., et al. 1999. Allergen-induced IL-9 directly stimulates mucin transcription in respiratory epithelial cells. J. Clin. Invest. 104:1375-1382.

22. Li, J.D., et al. 1998. Activation of NF-kappaB via a Src-dependent Ras-MAPK-pp90rsk pathway is required for Pseudomonas aeruginosa-induced mucin overproduction in epithelial cells. Proc. Natl. Acad. Sci. U. S. A. 95:5718-5723.

23. Hauber, H.P., et al. 2003. Increased expression of interleukin-13 but not interleukin-4 in cystic fibrosis patients. J. Cyst. Fibros. 2:189-194.

24. Miotto, D., et al. 2003. Interleukin-13 and -4 expression in the central airways of smokers with chronic bronchitis. Eur. Respir. J. 22:602-608.

25. Kuperman, D.A., et al. 2005. Dissecting asthma using focused transgenic modeling and functional genomics. J. Allergy Clin. Immunol. 116:305-311. 